\title{
Medicina, luz e fígado: uma breve reflexão de suas conexões
}

\section{Medicine, light and liver: a brief reflection of their connections}

\author{
Fabrícia Teixeira Mumic ${ }^{1}$, Orlando Castro e Silva ${ }^{2}$
}

"Esse é o caminho mais belo que uma teoria física pode assumir: quando ela abre caminho para uma teoria mais ampla, sem perder seu carácter individual." (Albert Einstein)

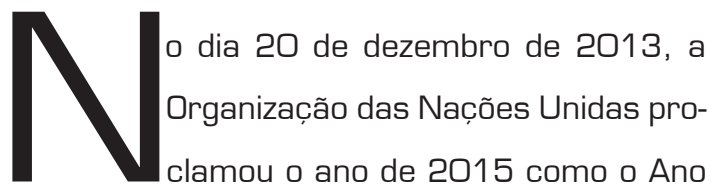
Internacional da Luz e das Tecnologias baseadas em Luz (International Year of Light and Light-based Technologies - IYL 2015), com o objetivo de ressaltar a importância da luz e das tecnologias ópticas na promoção do desenvolvimento sustentável e como ferramenta para os desafios mundiais nas áreas de energia, educação, agricultura, comunicação e saúde. ${ }^{1}$

As primeiras publicações a respeito da natureza da luz remontam de 1000 anos (1015), com o Tratado de sete volumes sobre óptica "Kitab al-Manazir" ["Book of Optics], escrito pelo cientista árabe "Ibn alHaytham", o qual proporcionou grandes contribuições para a compreensão da visão, óptica e luz. 1,2
A luz continuou sendo intrigante objeto de pesquisa para os que buscavam descobrir sua natureza. Em 1815, o físico francês Augustin-Jean Fresnel propôs o comportamento ondulatório da luz, seguido pelo físico e matemático britânico Maxwell que, em 1864 , propôs a teoria eletromagnética da luz.

Todos estavam convencidos de que a luz era composta de ondas eletromagnéticas, até que, em junho de 1905, Eistein publicou seu trabalho sobre o efeito fotoelétrico, apresentando seu estudo sobre a natureza dual da luz, onda-partícula. ${ }^{3}$ Assim, a partir da teoria fotoelétrica, foi possivel compreender o funcionamento das fontes de luz em suas mais diversas formas, como laser e LED, de forma a aplicá-los em múltiplos campos, desde físicos até a área da saúde.

A história da humanidade está repleta de exemplos nos quais a luz exerceu um papel importante na medicina. Recentemente, essa ideia tem sido fortalecida, originando um novo campo que combina ciências da saúde, física e engenharia, em uma conexão definida como biofotônica. 0 mais importante de-

1. Acadêmica do terceiro ano e aluna de Iniciação Científica da FMRP-USP.

2. Editor-chefe, Revista Medicina. Prof.Titular do Departamento de Cirurgia e Anatomia da Faculdade de Medicina de Ribeirão Preto, Universidade de São Paulo (FMRP-USP). 
senvolvimento da biofotônica foi transformar fontes de luz, como lasers e LED, em ferramentas acessiveis para pesquisa e aplicações, propiciando vasto campo de possibilidades tanto para o diagnóstico quanto para a terapêutica de inúmeras doenças. ${ }^{4,5}$

Lasers e LEDs são dispositivos incríveis que emitem luz com propriedades especiais. Enquanto lasers são coerentes e preservam a colimação durante a propagação, a luz originada do LED não é nem coerente nem colimada, e abrange um leque muito mais amplo de comprimentos de onda. No entanto, ambos estão sendo usados para diferentes tipos de aplicações relacionadas com diagnóstico e tratamento de doenças ou mesmo bioestimulação da regeneração tecidual. Esses avanços da biofotônica surgem como resposta à demanda de desenvolvimento de novas possibilidades para, seguindo a realidade econômica, produzir novos tratamentos, técnicas de diagnóstico e de prevenção. 4,5,6

O laser, em especial, tem sido usado como fonte luminosa de excitação para diagnóstico de doenças, como alternativa na intervenção cirúrgica e como parte de terapias modernas, tal qual a terapia fotodinâmica. Por outro lado, o LED dificilmente pode ser usado como dispositivo de corte, mas tem ampla aplicação como fonte de bioestimulação e terapia fotodinâmica. ${ }^{6}$

Nos últimos anos diversos estudos, através do uso de eficientes fontes de luz como laser e Light emitting diodes (LED), têm demonstrado a interação luz-tecido e suas diversas aplicações. Em especial, nossa equipe concentra pesquisas na interação luz-fígado e suas aplicações para promover a regeneração hepática após hepatectomias parciais, detectar alterações no fígado, como esteatose e hepatocarcinoma, avaliar o metabolismo hepático durante o transplante de fígado e diagnosticar e tratar alterações hepatocelulares. 5,6

O fígado é um órgão complexo que apresenta inúmeras propriedades, desde a característica de regeneração até suas funções no metabolismo, redistribuição de nutrientes como aminoácidos, gorduras, carboidratos, vitaminas e proteínas, síntese, desintoxicação (eliminação de xenobióticos e substâncias desnecessárias ao organismo], conversão de amônia em ureia e excreção de bile. ${ }^{7,8,9}$

A interação entre o laser e o fígado pode resultar em três possibilidades: estimulação, diagnóstico e uso terapêutico. Os estudos da nossa equipe visam a estimulação e a terapêutica, aumentando a regeneração hepática além de seus valores naturalmente alcançados, preservando a vitalidade funcional e metabólica do fígado. E, experimentalmente, a luz tem se apresentado como instrumento eficiente para bioestimular o crescimento hepático após ressecções parciais ou transplantes de fígado. 9-17

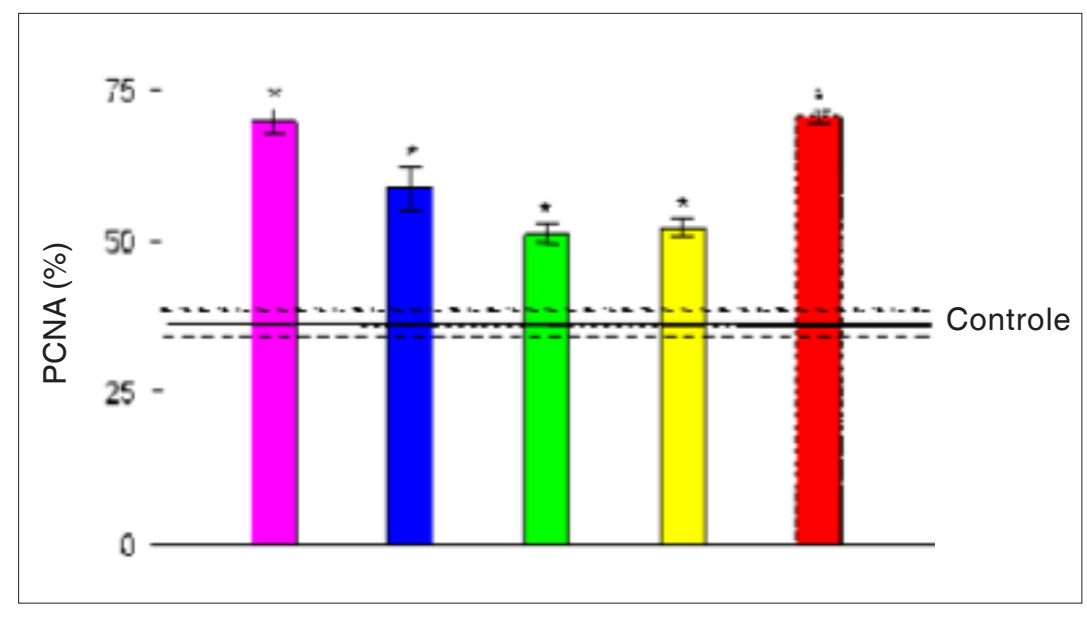

Figura 1: Regeneração hepática em resposta a estimulação do laser nos respectivos comprimentos de onda de luz visivel. ${ }^{14}$ 
Agindo no nível atômico-molecular do metabolismo celular hepático, a luz abriu caminho para intrigantes novas observações. Laser visivel de baixa intensidade está sendo usado para ativar a replicação do DNA mitocondrial ${ }^{6,18}$ e promover efeito estimulatório na regeneração celular (figura 1). 6,14,19,20 Algumas evidências de nossos experimentos mostraram que, após a aplicação da luz, são observados no fígado um aumento da respiração mitocondrial e da taxa de mitoses celulares, sem importantes alterações no metabolismo celular (figura 2). 5,6,13 Além disso, outras pesquisas de nosso grupo mostraram que após 24 horas do tratamento com o laser, há um aumento drástico da produção de ATP por miligrama de proteína mitocondrial, sem que o mesmo seja observado após longos intervalos de tempo (48 e 72 horas após a hepatectomia parcial], os quais apresentaram níveis semelhantes ao tecido não irradiado, o que mostra que a estimulação do laser altera somente os estágios iniciais do processo de regeneração hepática. Os mesmos resultados foram observados para atividade e consumo de oxigênio basal na mitocôndria. 5,6

Paralelamente ao efeito bioestimulante do laser, estudos mostraram que o LED promoveu regeneração hepática em ratos após hepatectomia parcial, sendo a que proliferação celular obtida com o laser e o LED foram similares, o que sugere que a irradiação de LED promove estimulação de efeitos biológicos durante os estágios iniciais da regeneração hepática e que o LED é tão efetivo quanto o laser, independente da coerência, divergência e cromaticidade. 20

Além do seu papel na regeneração hepática, a luz pode ser usada na forma de Terapia Fotodinâmica (PDT) como modalidade de tratamento para câncer. A Terapia Fotodinâmica consiste na combinação das propriedades das substâncias fotosensitivas com a citotoxicidade de seus subprodutos, de forma que, após a administração da droga seletiva para o tumor alvo, uma fonte de luz las principais fontes utilizadas atualmente são lasers, mas pesquisas mostraram equivalência entre laser e LED para essas aplicações] é usada para iluminar o tecido, iniciando uma reação fotoquímica que gera espé-

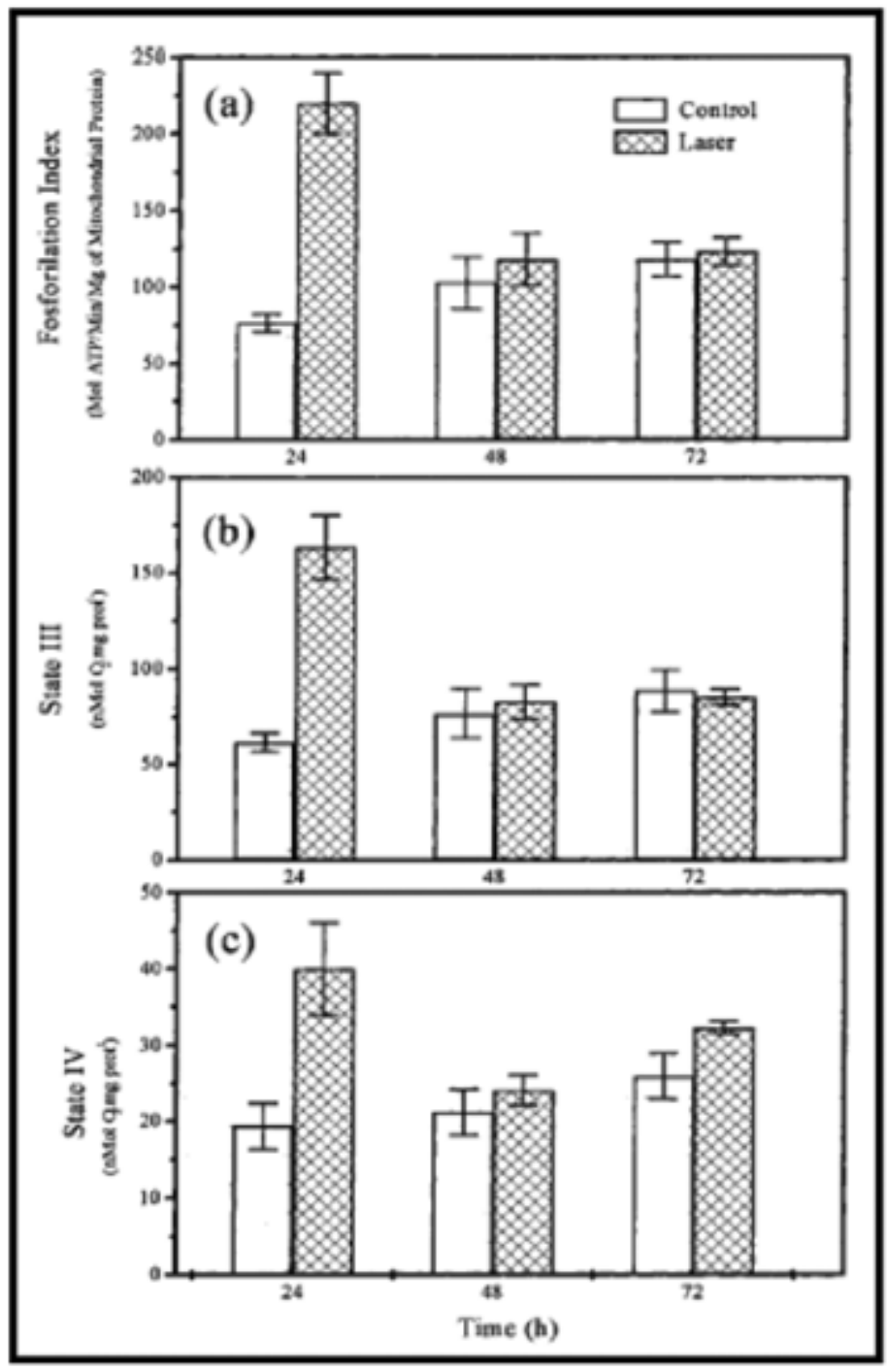

Figura 2: Medidas da atividade de oxidação e fosforilação mitocondriais. a: Índice de fosforilação. b: Estado III (consumo basal mitocondrial de oxigênio). c: Estado IV (atividade mitocondrial estimulada com succinato]. Nessas três medidas, há significante aumento no grupo tratado com laser em comparação com o grupo controle 24 horas após a hepatectomia. ${ }^{6}$ 
cies oxidativas extremamente citotóxicas, resultando finalmente na morte do tecido que continha a substância fotosensitiva e que foi iluminada. A seletividade ajuda na prevenção da morte das células normais, fator importante para a recuperação do tecido. Além disso, o dano necessário para matar uma célula cancerígena é menor do que o necessário para matar uma célula normal. No fígado, a PDT é utilizada com certa restrição, pois no mesmo a seletividade entre as células tumorais e as células hepáticas não é tão eficiente. No entanto, acredita-se que com os recentes avanços, essa dificuldade possa ser superada. ${ }^{6}$

As aplicações modernas da luz como ferramenta para diagnóstico e tratamento de disfunções hepáticas parecem muito realistas e aplicáveis clinicamente. Através da análise da espectroscopia, é possível detectar lesões por análises ópticas, reduzindo a necessidade de procedimentos invasivos e fornecer informações importantes para o diagnóstico de lesões dúbias. ${ }^{6}$ Nossa equipe, do Departamento de Cirurgia e Anatomia da Faculdade de Medicina de Ribeirão Preto - Universidade de São Paulo em parceria com o Instituto de Física de São Carlos da citada Universidade, almeja novas descobertas e continua com as pesquisas explorando a interação entre a luz e o fígado, com grandes ambições para o ano de 2015, o Ano Internacional da Luz e das Tecnologias baseadas em Luz (International Year of Light and Light-based Technologies - IYL 2015].

\section{Referências}

1. Site oficial Ano Internacional da Luz e das Tecnologias baseadas em Luz (International Year of Light and Light-based Technologies - IYL 2015) - http://www.light2015.org [ Acesso em: 10 de fevereiro de 2015].

2. Abdelhamid I. Sabra. Ibn al-Haytham - Brief life of an Arab mathematician: died circa 1040 - Harvard Magazine - September-October 2003.

3. Bertolotti, Mario (2005), The history of laser. Institute of Physics Publishing Bristol and Philadelphia.
4. Bagnato VS, Kurachi C, Castro-e-Silva O. New perspectives for optical techniques in diagnostic and treatment of hepatic diseases. Acta Cir Bras. 2010; 25: 214-6.

5. Castro e Silva O, Bagnato VS, Kurachi C, Ferreira J, Sankarankutty AK, Zucoloto S. Novas Tecnologias Fotônicas para o Tratamento e Diagnóstico de Doenças Hepáticas. In: Bagnato VS. Novas técnicas ópticas para áreas de saúde. São Paulo: Editora Livraria da Física, 2008. p. 229-39.

6. Bagnato VS, Kurachi C, Ferreira J, Sankarankutty AK, Zucoloto $S$, Castro e Silva O. New photonic Technologies for the treatment and diagnosis os hepatic diseases: An overview of the experimental work performed in collaboration, between Physics Institute of São Carlos and Ribeirão Preto Faculty of Medicine os University of São Paulo. Acta Cir Bras. 2006;21 Suppl 1

7. Fausto N. Liver regeneration. J Hepatol. 2000;32:19-31.

8. Mortesen KE, Revhaug A. Liver regeneration in surgical animal models - A historical perspective and clinical implications. Eur Surg Res. 2011;46:1-18

9. Miyaoka Y, Miyajima A. To divide or not divide: revisiting liver regeneration. Cell Div. (Lond.). 2013; 8:1-12

10. Castro e Silva Jr O; Silveira MRG. Fígado e luz. Uma combinação precisa, da mitologia à medicina. Medicina (Ribeirao Preto. Online). 2013; 46: 318-21, ISSN 2176-7262.

11. Melo CA, Lima AL, Brasil IR, Castro e Silva Jr O, Magalhães DV, Marcassa LG, Bagnato VS. Characterization of light penetration in rat tissues. J Clin Laser Med Surg. 2001; 19:175-9.

12. Melo GB, Silva RL, Melo VA, Lima SO, Antoniolli AR, Castro e Silva $T$, et al. Enhancement of liver re- generation by the association of Hyptis pectinata with laser therapy. Dig Dis Sci. 2005;50:949-54.

13. Castro e Silva Jr O, Zucoloto S, Bagnato VS, Marcassa LG, Menegazzo LAG, Granato RG. Laser enhancement in hepatic regeneration for partially hepatectomized rats. La- sers Surg Med. 2001;29:73-7.

14. Castro e Silva O Jr, Zucoloto S, Marcassa LG, Marcassa J, Kurachi C, Melo CA, et al. Spectral response for enhancement in hepatic regeneration for hepatectomized rats. Lasers Surg Med. 2003; 32: 50-3.

15. Ferraz RC, Ferreira J, Menezes PF, Sibata $\mathrm{CH}$, Castro e Silva O Jr, Bagnato VS. Determination of threshold dose of photodynamic therapy to measure superficial necrosis. Photomed Laser Surg. 2009; 27:93-9.

16. Castro e Silva O, Prozillo LC, Potenciano O, Mucillo G, Zucoloto $\mathrm{S}$. The Effect Of Low-Energy He-Ne Laser Irradiation On Liver Regeneration. Res Sur. 1991; 3:192-3.

17. Castro e Silva O, Ramalho LZ, Zilio S, Bagnato VS, Granato RG, et al. Aspectos Basicos da Regeneracao Hepatica e O Papel da Luz Laser e Convencional Como Agentes Estimulantes. Acta Cir Bras. 1996;11:14-6

18. Vacca RA, Marra E, Quagliariello E, Greco M. Activation of DNA mitocondrial replication by $\mathrm{He}-\mathrm{Ne}$ irradiation. Biochem Biophys Res Commun. 1993;95:704:9.

19. Karu TI, Pyatibrat LV, Kalendo GS, Serebriakov NG. Changes in the amount of ATP in Hela cells under the action of $\mathrm{He}-\mathrm{Ne}$ laser radiation. Bull Eksp Biol Med. 1993;115:617-8

20. Castro-e-Silva T, Castro-e-Silva O, Kurachi C, Ferreira J, Zucoloto S, Bagnato VS. The use of light-emitting diodes to stimulate mitochondrial function and liver regeneration of partially hepatectomized rats. Braz J Med Biol Res [online]. 2007; 40: 1065-9. [cited 2015-02-22]. 